\title{
A Hybrid Vortex Method for Two-Dimensional Flow Over Tube Bundles
}

\author{
Walter P. Wolfe (wpwolfe@sandia.gov) \\ James H. Strickland (jhstric@sandia.gov) \\ Engineering Sciences Center \\ Sandia National Laboratories* \\ P.O. Box 5800 \\ Albuquerque, NM 87185-0836, USA
}

\begin{abstract}
A hybrid vortex method is presented for computing flows about objects that accurately resolves the boundary layer details while keeping the number of free vortices at a reasonable level. The method uses a wall layer model close to the body surface and discrete vortex blobs in the free wake. Details of the wall layer implementation are presented, and results of sample calculations are compared with known analytical solutions and with calculations from other vortex codes. These results show that the computed boundary layer details are accurate to approximately 0.3 percent of analytical solutions while using three orders of magnitude fewer vortices than other vortex simulations.
\end{abstract}

\section{Introduction}

In the present work we consider a hybrid vortex method for calculating the two-dimensional fluid flow over bluff bodies, such as tube bundles. The motivation for this work arises from the need to develop a predictive computational capability for the accurate modeling of the coupled fluid and structural dynamics of highly flexible structures. Engineering applications for such a capability include deepwater drilling and production risers, tubular heat exchangers, submarine-towed sensor arrays, and flow around parachute ribbons.

We have had good success using gridless vortex methods to model unsteady, high Reynolds number flow over various engineering devices. These simulations have included the prediction of performance of, and fluid forces on, vertical-axis wind turbines [22, 23]; the calculation of wake development behind aircraft [8] and submarines [20]; and the prediction of flow over simple axisymmetric parachute shapes [18]. In contrast to conventional grid-based computational fluid dynamics methods, the gridless vortex method is noniterative, artificial viscosity is not required for stability, numerical diffusion is minimal, and moving boundaries may be easily treated. A salient feature of the method is that the computational domain only includes those regions where there is vorticity. For higher Reynolds numbers this domain is increasingly reduced, since vorticity occupies a smaller and smaller fraction of the fluid volume.

Methods for transporting the vorticity from the surfaces of bodies during the computation can be grouped into a few general categories. The first is the injection of vorticity at prescribed surface injection points (Figure 1). Examples of these methods are cited by Sarpkaya [16]. While these methods are extremely efficient since they minimize the number of vortices injected into the flowfield, the user must guess a priori the flow separation points, and the method cannot account for secondary flow separation.

A second category can be called blanket surface flux. In these methods large numbers of very small discrete vortex elements are placed next to the surface at each time step (Figure 2). Examples include the work of Koumoutsakos [12], Lin et al. [14], and Shih et al. [17]. While these methods can accurately compute flow separation points, they also have some disadvantages. One is that a large number of vortex elements must be used. The vortex diameters must be some fraction of the boundary layer thickness, and for high Reynolds number flows, the required number of vortex blobs becomes prohibitive. Another is that unless

\footnotetext{
* Sandia is a multiprogram laboratory operated by Sandia Corporation, a Lockheed Martin Company, for the U.S. Department of Energy under contract No. DE-AC04-94AL85000.
} 
the element density close to the surface is very high, the details of the boundary layer are not accurately modeled.

A third approach being developed by Marshall and Grant [15] alleviates some of these problems by using anisotropic blobs that have thicknesses that are a fraction of the boundary layer thickness and lengths that are on the order of the surface panel length.

The best approach is to use a wall-layer model (Figure 3), which produces a robust algorithm that is compatible with discrete vortex methods while at the same time yielding good resolution of the vorticity/velocity field near the wall. Outside of the wall layer the vortex elements are discrete circular blobs with diameters on the order of the surface panel length, $h$. The vortex elements defined in each region are able to smoothly interact with the elements of the other region and transition from one region to the other. The details of the wall layer, the interactions between the wall layer and the free vortex blobs, and the transition of vorticity into and out of the wall layer are given below. A different implementation of the wall layer concept has also been used by Chou [5]. The present method is intended to yield good resolution of the diffusion and convection processes close to the body without generating any free vortex blobs that are smaller than $\mathrm{O}(h)$.

\section{Wall Layer Description}

The wall layer is a thin meshed region adjacent to the wall within which circulation is modeled using quadrilateral elements (mesh cells) that are elongated in the tangential direction as compared with their thickness normal to the wall (Figure 4). It is composed of three distinct sublayers, $0<y<h, h<y<2 h$, and $2 h$ $<y<3 h$, where $y$ is the distance normal to the wall. Within the first sublayer, $0<y<h$, all circulation is contained within the mesh cells. The second sublayer, $h<y<2 h$, is a transition region where circulation can exist either within the mesh cells or as discrete vortex elements. When vorticity has been convected and diffused outward from the wall into the outer regions of Sublayer 2, it is converted to a discrete vortex blob. This conversion conserves the total circulation and the first and second moments of vorticity. The third sublayer, $2 h<y<3 h$, is an overflow region designed to catch any vorticity that "leaks" out of the top of the wall layer during a time step before a discrete vortex can be constructed. Any vorticity that enters Sublayer 3 is immediately converted into a vortex blob.

The mesh within the wall layer is defined using orthogonal, curvilinear coordinates. This allows one to march outward from the wall and obtain the velocity field from the local vorticity field without having to explicitly consider the vorticity in the wake. Diffusion and convection of vorticity is accomplished using a Lagrangian-Eulerian scheme. At each time step the grid moves with the vorticity during diffusion and convection. The solution is then mapped back onto the original orthogonal grid. Circulation is explicitly conserved during each step in this process. After the remap step vorticity is generated at the wall to satisfy the prescribed boundary conditions.

The linear nature of vorticity allows us to split the wall-layer vorticity into its positive and negative components. Each component is diffused and remapped independently. Both components are convected with the total convection velocity. This splitting greatly improves the numerical stability of the code when computing problems that have both signs of vorticity. The equations for vorticity generation, diffusion, convection, and remapping are developed in the following sections.

\section{Convection Velocity}

The equations used to compute the convection velocity within the wall layer are derived from the definition of vorticity in terms of the curl of the velocity field, $\vec{\omega}=\nabla \times \vec{V}$, and the continuity equation, $\nabla \bullet \vec{V}=0$. Expressing these equations in orthogonal, curvilinear coordinates; combining them with the derivative chain rule; and restricting the integration to lines of constant $\xi$ we obtain

$$
V_{\xi}=V_{\xi_{w}}+\int_{w}^{p}\left(-\omega h_{\eta}+\frac{1}{h_{\xi}} \frac{\partial}{\partial \xi}\left(h_{\eta} V_{\eta}\right)-\frac{V_{\xi}}{h_{\xi}} \frac{\partial h_{\xi}}{\partial \eta}\right) d \eta
$$


and

$$
V_{\eta}=V_{\eta_{w}}+\int_{w}^{p}\left(-\frac{1}{h_{\xi}} \frac{\partial}{\partial \xi}\left(h_{\eta} V_{\xi}\right)-\frac{V_{\eta}}{h_{\xi}} \frac{\partial h_{\xi}}{\partial \eta}\right) d \eta,
$$

where $\xi$ and $\eta$ are orthogonal, curvilinear coordinates (at the body's surface $\xi$ is parallel to the wall, and $\eta$ is normal to the wall); $h_{\xi}$ and $h_{\eta}$ are the transformation scale factors defined as $h_{\xi}=|\partial \vec{r} / \partial \xi|$ and $h_{\eta}=|\partial \vec{r} / \partial \eta|$; and $\vec{r}$ is the position vector. Equations (1) and (2) can be integrated from the wall, $w$, where the velocity is known, to obtain the values of $V_{\xi}$ and $V_{\eta}$ at any point, $p$, in the wall layer.

\section{Diffusion Velocity}

Diffusion of vorticity can be accomplished by one of several means. The diffusion method we use is based on the diffusion velocity concept of Kempka and Strickland $[11,21]$. The diffusion velocity is specified such that the circulation within a given boundary remains constant with time if that boundary moves at the diffusion velocity plus the local fluid velocity. In Reference [11] the diffusion velocity, $\vec{u}_{d}$, is shown to be

$$
\vec{u}_{d} \times \vec{\omega}=-v \nabla \times \vec{\omega} .
$$

For two-dimensional flow this reduces to

$$
\vec{u}_{d}=-\frac{v}{\omega} \nabla \omega .
$$

In orthogonal, curvilinear coordinates this equation becomes

$$
\vec{u}_{d}=-\frac{v}{\omega}\left\{\frac{1}{h_{\xi}} \frac{\partial \omega}{\partial \xi} \hat{\xi}+\frac{1}{h_{\eta}} \frac{\partial \omega}{\partial \eta} \hat{\eta}\right\},
$$

where $\hat{\xi}$ and $\hat{\eta}$ are the unit vectors.

\section{Vorticity Generation and Diffusion into the Wall Layer}

Vorticity is generated at the wall at each time step in order to satisfy the velocity boundary conditions. There are several ways to obtain formulations aimed at the satisfaction of these boundary conditions. However, some methods will lead to unsatisfactory results. Kempka et al. [10] provide a discussion of some of the problems associated with satisfying velocity boundary conditions and present a formulation in which the no-slip boundary condition is satisfied explicitly, and the normal boundary condition is satisfied implicitly.

In the present work the normal-velocity boundary condition is satisfied explicitly by placing a vortex sheet on the body surface. The no-slip boundary condition is used to split the calculated vortex sheet into two vortex sheets that lie at distances $\pm \varepsilon$ on either side of the body surface. This method is somewhat similar to one outlined by Strickland [18] in which a stream function formulation was used to satisfy velocity boundary conditions for axisymmetric flow over thin shells.

Assuming that the diffusion at the wall is predominately one-dimensional and switching to Cartesian coordinates for notational simplicity, Equation (5) becomes

$$
\left.u_{d}\right|_{w}=-\left.\frac{v}{\omega} \frac{\partial \omega}{\partial y}\right|_{w},
$$


where $y$ is measured normal to the wall. The flux of the circulation $\gamma$ per unit length of $x$ across the wall during a computational time step is simply the product of the diffusion velocity at the wall and the added vorticity, or

$$
\left.\frac{\partial \gamma}{\partial t}\right|_{w}=-\left.v \frac{\partial \omega}{\partial y}\right|_{w}
$$

which means that the vorticity gradient at the wall is

$$
\left.\frac{\partial \omega}{\partial y}\right|_{w}=-\left.\frac{1}{v} \frac{\partial \gamma}{\partial t}\right|_{w} .
$$

We have examined several numerical models for diffusing the vorticity into the flow at the wall. In a recent paper by Koumoutsakos, Leonard, and Pepin [13] concerning vorticity generation at a wall, an algorithm for updating the particle strengths in a particle strength exchange (PSE) method was presented for finite panels with curvature. In the context of the present one-dimensional model, the vorticity that would be introduced into the domain from the wall during a time $\Delta t$ is

$$
\Delta \omega=\omega^{t+\Delta t}-\omega^{t}=\frac{\gamma \exp \left(-\frac{y^{2}}{4 v \Delta t}\right)}{\sqrt{\pi v \Delta t}},
$$

where $\omega^{t+\Delta t}$ is the vorticity at the time $t+\Delta t$, and $\omega^{t}$ is the vorticity at time $t$. We note that Equation (9) is identical to the exact solution for an impulsively started flat plate [6]. Adding all of the circulation to the first wall layer element at the beginning of the time step is roughly equivalent to using Equation (9) at the end of the time step.

Since the diffusion equation is linear, we may obtain the vorticity distribution associated with the flux from the wall at the end of time $\Delta t$ by superimposing the impulsive solutions via the following convolution integral,

$$
\Delta \omega=\int_{0}^{\Delta t} \frac{\partial \gamma}{\partial \tau} \frac{\exp \left(-\frac{y^{2}}{4 v(\Delta t-\tau)}\right)}{\sqrt{\pi v(\Delta t-\tau)}} d \tau
$$

Assuming that $\partial \gamma / \partial \tau$ is constant over the time interval $\Delta t$ and defining $y^{+} \equiv y / \sqrt{v \Delta t}$, Equation (10) can be integrated numerically to obtain the curve labeled as "Exact" in Figure 5. The resulting distribution can be fitted with a curve given by

$$
\Delta \omega^{+} \equiv \Delta \omega \sqrt{\frac{\nu}{\Delta t}} / \frac{\partial \gamma}{\partial t}=1.131 e^{-q},
$$

where

$$
q=0.884 y^{+}+0.161 y^{+2}
$$

For comparison the distribution given by a single impulse at the mid-time is also plotted in Figure 5, identified in the legend by $0.5 \Delta t$. The major difference in the two curves is their distributions near the wall. From Equation (8) it is seen that the slope of the $\left(\Delta \omega^{+}, y^{+}\right)$curve at the wall must be equal to minus one, 
not zero, as is given by the impulsive start representation. We note that the proper slope is obtained when using Equation (11). For simulations where $\Delta t \rightarrow 0$, the global vorticity distribution will tend to be the same for the various representations of the wall flux distribution. On the other hand, we have found that one has to sometimes take much smaller time steps when using the impulsive representation in order to obtain satisfactory distributions near the wall. We therefore recommend the use of Equation (11).

\section{Mesh Movement and Remapping}

Vorticity within the wall layer is convected and diffused at each time step using a Lagrangian-Eulerian scheme. In the Lagrangian step each mesh node is moved a distance equal to the sum of the local diffusion and convection velocities times the time step $\Delta t$. The total circulation within each grid cell is held constant during this movement. In the Eulerian step the vorticity field is mapped back onto the original orthogonal mesh using an implementation of the second-order, conservative, remapping scheme of Dukowicz and Kodis [7]. A first-order remapping scheme is much too diffusive for practical use. A complete description of this scheme is beyond the scope of this work, and the reader is referred to [7] for a complete derivation and discussion of the method.

\section{Discrete Vortex Blobs}

After each remapping step the circulation with the wall layer is examined for possible formation of discrete vortex blobs. Discrete blobs are formed whenever either one of two criteria are met: (1) If an outer cell of the second wall sublayer contains circulation after the remapping step, then all of the circulation within the cells of that mesh column and within that sublayer is converted into a discrete transition blob; or (2) if any vorticity has moved into the third wall sublayer, then all vorticity within that mesh column and within that sublayer is also converted. The conversion process preserves the total circulation and the first and second moments of the converted vorticity. The first moment determines the blob's location. The second moment determines the blob's core radius. The converted vorticity is removed from the wall layer. Positive and negative vorticity are treated separately.

A discrete vortex blob is classified as a free or transition blob depending upon its location. If a blob's center is within wall Sublayer 2, that blob is a transition blob. Once the blob has moved outside Sublayer 2, it is a free vortex blob. This classification is primarily for code bookkeeping. The only meaningful difference between transition and free blobs is the means for computing their convective velocity. The convective velocity for free blobs is computed from the Biot-Savart law using a Greengard-Rokhlin fast solver [19, 3]. For transition blobs the convective velocity is interpolated from the wall layer convective velocities. Blobs can move freely into and out of the wall layer, and their classification is changed accordingly. If a blob moves into wall Sublayer 1, it is absorbed into the wall layer.

In order to compute convective velocities using Equations (1) and (2), all vorticity along the integration path must be included. For these calculations all blobs that overlap the wall layer are mapped into the layer. Because a Gaussian distribution does not have finite support, the blob distribution for this mapping is assumed to be a right-circular cone. The conical distribution is computed by preserving the total circulation and the first and second moments of the Gaussian blob's vorticity.

\section{Example Calculations}

In order to verify that our wall layer implementation accurately models the fluid mechanics in the vicinity of a solid boundary, we compare the calculated results with two classical fluid flows that have known solutions: (1) flow over a semiinfinite flat plat (Blasius flow) and (2) flow next to an oscillating flat plate (Stokes' second problem). Since the purpose of these calculations was to verify the wall layer coding, no free vortices were used. All of the vorticity was contained within the wall layer. The Blasius solutions used 45 cells normal to the plate. The oscillating plate solution used 30 cells normal to the plate. For both calculations the maximum error in computed vorticity and total velocity is on the order of 0.3 percent.

Figure 6 compares the calculated vorticity distribution normal to the wall with the Blasius distribution. The variables have been nondimensionalized to remove the dependence on Reynolds number. $U_{0}$ is the 
freestream velocity. While we only show results for one calculation, we made several calculations at different local Reynolds numbers. All gave the same nondimensional results. Figure 7 compares the tangential velocity profile, and Figure 8 compares the normal velocity profile for the same calculation. All show excellent results.

Figure 9 compares the vorticity distributions normal to a flat plate undergoing sinusoidal motion at three different phase angles during a cycle. Sufficient time has passed so that the solution has stabilized to a periodic solution. $U_{0}$ is the maximum velocity of the plate; $n$ is the plate's oscillation frequency. Figure 10 shows the tangential velocity profiles for the periodic solution at these same phase angle. Figure 11 shows the vorticity distribution during the initial start-up phase at one phase angle. The flow stabilizes after approximately five cycles. Again all comparisons show excellent agreement.

Figure 12 compares the results from our current vortex-based implementation with calculations by Chang and Chern [4] and Koumoutsakos [4] for the drag coefficient of an impulsively started circular cylinder during the first two diameters of travel. $C_{X}$ is nondimensionalized by the free stream dynamic pressure and the cylinder's cross-sectional area. The Reynolds number, based on cylinder diameter, for these calculations was 9500. Also shown is the theoretical result of Bar-Lev and Yang [1] for the first radius of travel. Although not an exact match, our calculations are in reasonably good agreement with the other results. The oscillations in the current simulation in the latter half of the run occur due to the formation of discrete vortex blobs. Numerical experimentation has shown that reducing the size of the surface panels gives a smoother force curve. The extremely large numbers of vortices used by Chang and Chern and by Koumoutsakos give a smoother force. It should be noted that the simulation of Koumoutsakos required over 500,000 vortices [12]. Our current simulation using the wall layer required less that 300 .

Figure 13 shows the computed wake behind an impulsively started circular cylinder for a Reynolds number based on cylinder diameter of 90,000. The computed side force and drag coefficients are shown in Figure 14 and Figure 15, respectively. Both coefficients are nondimensionalized by the free stream dynamic pressure and the cylinder's cross-sectional area. For this simulation the cylinder was modeled using 40 surface panels. We also made simulations using 20 and 80 surface panels. The simulations with 40 and 80 panels gave the same results. The simulation using 20 panels gave different results, indicating that the surface paneling and/or the wall layer mesh was too coarse. The time step for the simulation was chosen such that the body moves no more than one panel length during a time step. For this simulation, a time step of 0.05 was used.

After the impulsive start the flow remains reasonably axisymmetric for approximately four diameters of travel. After that time the wake asymmetries begin to appear. The von Karman vortex street is well developed during the last ten diameters of travel. The Strouhal number during the last ten diameters of travel, computed from the side force period in Figure 14, is approximately 0.22 , which compares favorably with the published value of 0.2 [2]. The average drag coefficient during the final five diameters of travel (Figure 15 ) is approximately 1.0. This is slightly lower than the more accepted number for subcritical flow of 1.17 [9].

\section{Summary and Conclusions}

We have presented a description of a hybrid computational fluid dynamics vortex scheme for computing flows about objects that accurately resolves the boundary layer details while keeping the number of free vortices in the calculation at a reasonable level. The scheme uses a wall layer model in which the flow in the vicinity of the body is modeled using a Lagrangian mesh. Circulation is treated as field variable within the mesh and is assigned to the mesh cells. Outside the wall layer the flow is modeled using discrete vortex blobs. Vorticity is able to smoothly transition between the wall layer and the free wake. Comparisons of the results of calculations using this scheme, with flows that have known solutions, indicate that the solutions are accurate to approximately 0.3 percent. Comparisons with other calculations for flow over an impulsively started circular cylinder show good agreement while using a factor of $10^{3}$ fewer vortices. 


\section{References}

1. Bar-Lev, M., and Yang, H. T., "Initial Flow Field Over an Impulsively Started Circular Cylinder," J. Fluid Mech., 72, pp. 625-647, 1975.

2. Blevins, R. D., Flow-Induced Vibration, Van Nostrand Reinhold, New York, pp. 47-48, 1990.

3. Carrier, J., Greengard, L., and Rokhlin, V., "A Fast Adaptive Multipole Algorithm for Particle Simulations," SIAM Journal on Scientific and Statistical Computing, Vol.9, No.4, pp. 669-696, July 1988.

4. Chang, C. C., and Chern, R. L., "A Numerical Study of Flow Around an Impulsively Started Circular Cylinder by a Deterministic Vortex Method," J. Fluid Mech., 233, pp. 243-263, 1991.

5. Chou, M., "Simulation of Slightly Viscous External Flow by a Grid-Particle Domain Decomposition Method," Computers \& Fluids, Vol. 24, No. 3, pp. 333-347, 1995.

6. Currie, I. G., Fundamental Mechanics of Fluids, McGraw-Hill, pp. 231-237, 1974.

7. Dukowicz, J. K., and Kodis, J. W., "Accurate Conservative Remapping (Rezoning) for Arbitrary Lagrangian-Eulerian Computations," SIAM J. Sci. Stat. Comput., Vol. 8, No. 3, pp. 305-320, May 1987.

8. Fullerton, T. L., Strickland, J. H., and Sundberg, W. D., "A Vortex Panel method for Calculating Aircraft Downwash on Parachute Trajectories," AIAA paper 91-0875, presented at the AIAA 11th Aerodynamic Decelerator Systems Technology Conference, April 1991.

9. Hoerner, S. F., Fluid-Dynamic Drag, Published by the Author, Vancouver, WA, pp. 3-15 - 3-17, 1965.

10. Kempka, S. N., Glass, M. W., Perry, J. S., and Strickland, J. H., "Accuracy Considerations for Implementing Velocity Boundary Conditions in Vorticity Formulations," Sandia National Laboratory Report SAND96-0583, March 1996.

11. Kempka, S. N., and Strickland, J. H., "A Method to Simulate Viscous Diffusion of Vorticity by Convective Transport of Vortices at a Non-Solenoidal Velocity," SAND93-1763, Sandia National Laboratories, Aug. 1993.

12. Koumoutsakos, P. D., "Direct Numerical Simulations of Unsteady Separated Flows Using Vortex Methods," Ph.D. Dissertation, California Institute of Technology, 1993.

13. Koumoutsakos, P., Leonard, A., and Pepin, F., "Boundary Conditions for Viscous Vortex Methods," J. Comput. Phy., vol. 113, pp. 52-61, 1994.

14. Lin, H., Vezza, M., and Galbriath, R. A. McD., "Discrete Vortex Method for Simulating Unsteady Flow Around Pitching Airfoils," AIAA Journal, Vol. 35, No. 3, pp. 494-499, March 1997.

15. Marshall, J. S., and Grant, J. R., "A Method for Determining the Velocity Induced by Highly Anisotropic Vorticity Blobs," J. of Computational Physics, 126, pp. 286-298, 1996.

16. Sarpkaya, T., "Vortex Element Methods for Flow Simulation," Advances in Applied Mechanics, Vol. 31, pp. 113-247, 1994.

17. Shih, C., Lourenco, L., Van Dommelen, L., Krothapalli, A., "Unsteady Flow Past an Airfoil Pitching at a Constant Rate," AIAA Journal, Vol. 30, No 5, pp. 1153-1161, May 1992.

18. Strickland, J. H., "A Prediction Method For Unsteady Axisymmetric Flow Over Parachutes," AIAA paper 93-1217, presented at the RAeS/AIAA 12th Aerodynamic Decelerator Systems Technology Conference, London, England, May10-13, 1993. Also, AIAA Journal of Aircraft, Vol. 31, No. 3, pp. 637643, May-June 1994.

19. Strickland, J. H., and Baty, R. S., "A Two-Dimensional Fast Solver for Arbitrary Vortex Distributions," Sandia National Laboratories Report SAND97-0880, April 1997. 
20. Strickland, J. H., and Kempka, S. N., "Modeling Wake Evolution Behind a Submarine: Two Dimensional Maneuvers," Sandia National Laboratories Report, SAND93-0040, April 1993.

21. Strickland, J. H., Kempka, S. N., and Wolfe, W. P., "Viscous Diffusion of Vorticity Using the Diffusion Velocity Concept," ESAIM Proceedings, Vol. 1, pp. 101-117, Aug. 1996. (http://www.emath.fr/Maths/Proc/Vol.1/index.htm)

22. Strickland, J. H., Webster, B. T., and Nguyen, T., "A Vortex Model of the Darrieus Turbine: An Analytical and Experimental Study," Journal of Fluids Engineering, Vol. 101, No. 4, pp. 500-505, 1979.

23. Touryan, K. J., Strickland, J. H. and Berg, D. E., "Electric Power From Vertical-Axis Wind Turbines (A Survey)," AIAA Journal of Propulsion and Power, Vol. 3, No. 6, pp. 481-493, Nov-Dec 1987. 


\section{Figures}

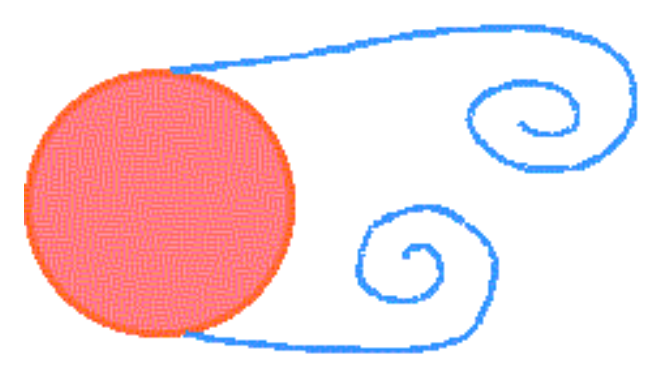

Figure 1. Prescribed Surface Injection Points

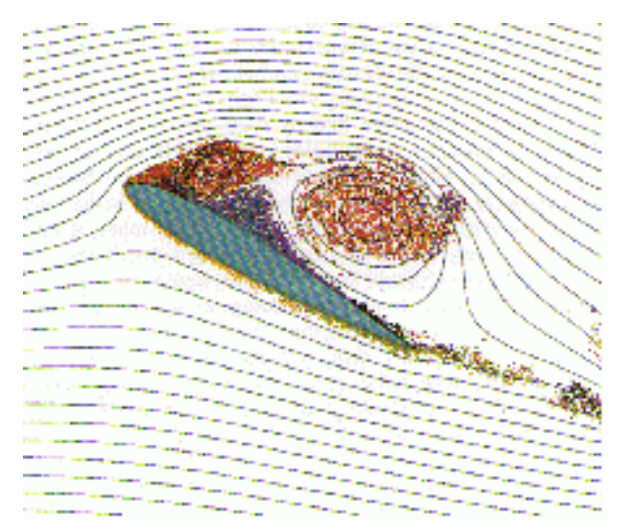

Figure 2. Blanket Surface Flux (from Ref. 17)

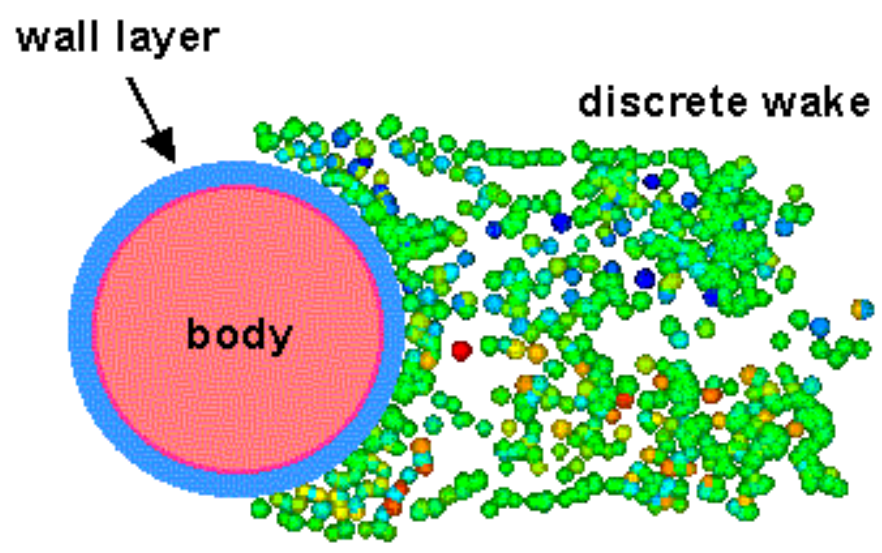

Figure 3. Wall Layer Model 


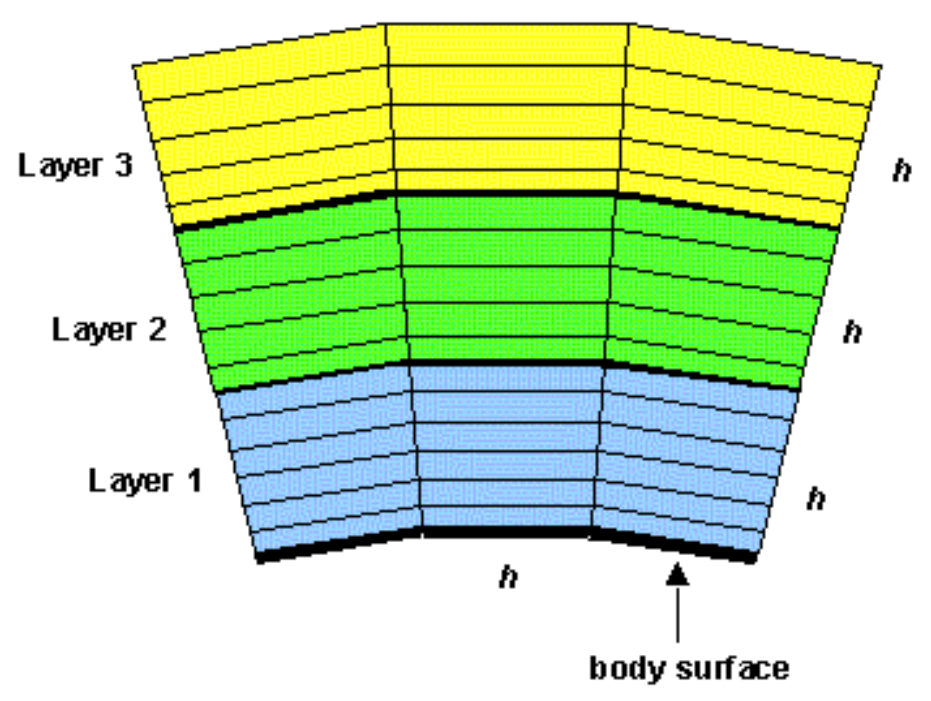

Figure 4. Wall Layer Details

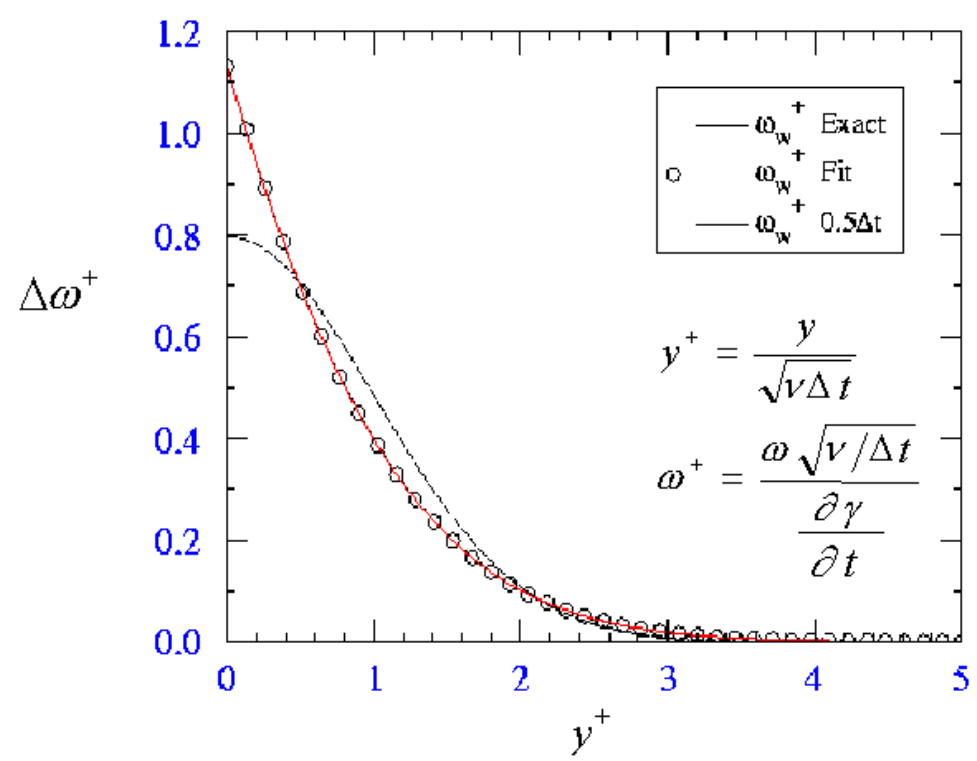

Figure 5. Distribution of Wall Vorticity 


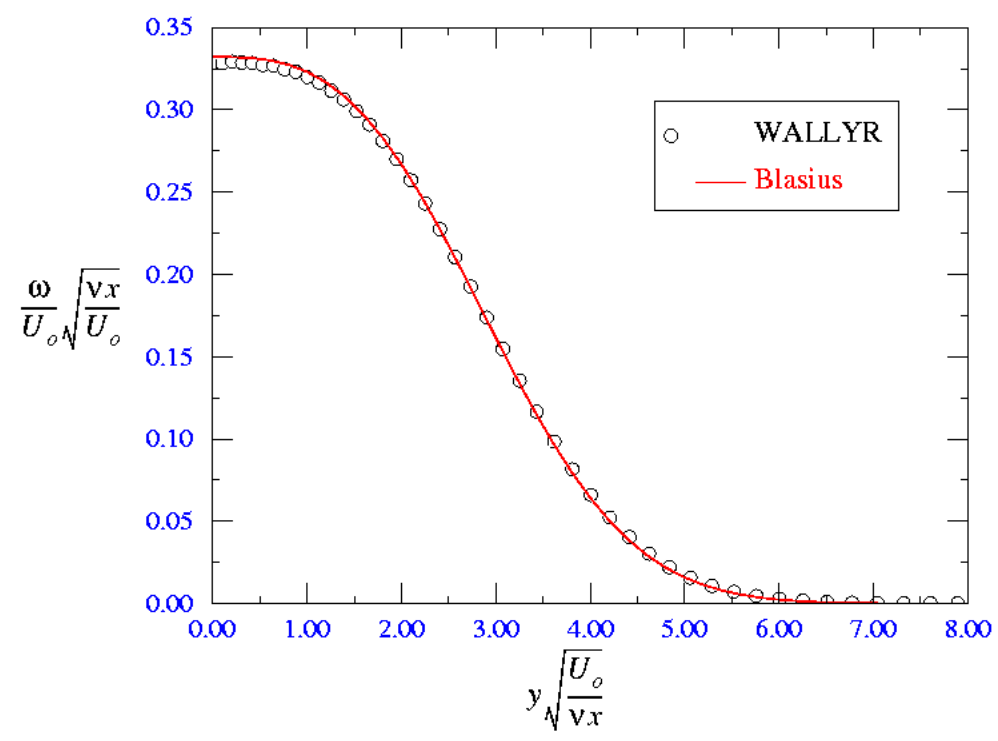

Figure 6. Vorticity Distribution for Blasius Solution

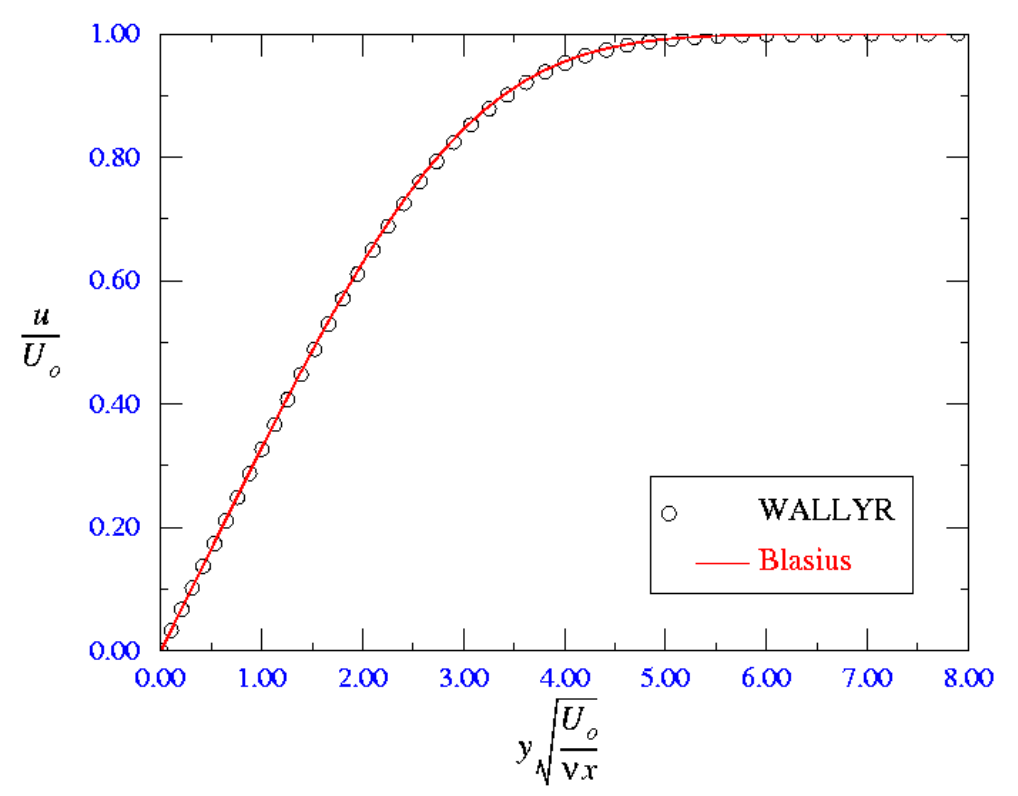

Figure 7. Tangential Velocity Profile for Blasius Solution 


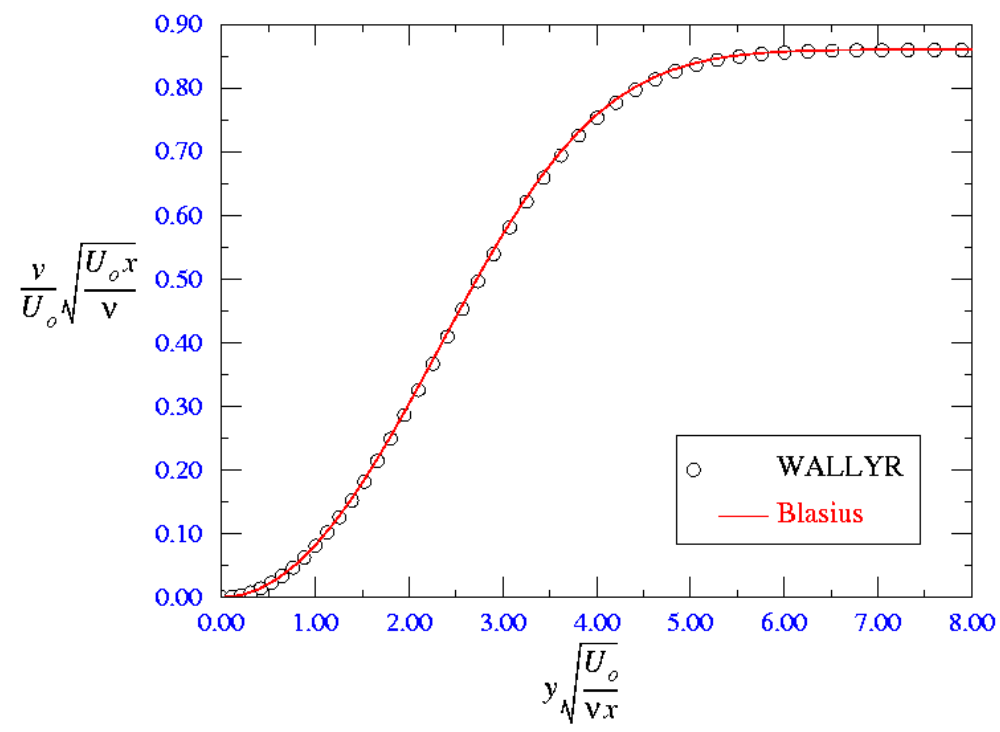

Figure 8. Normal Velocity Profile for Blasius Solution

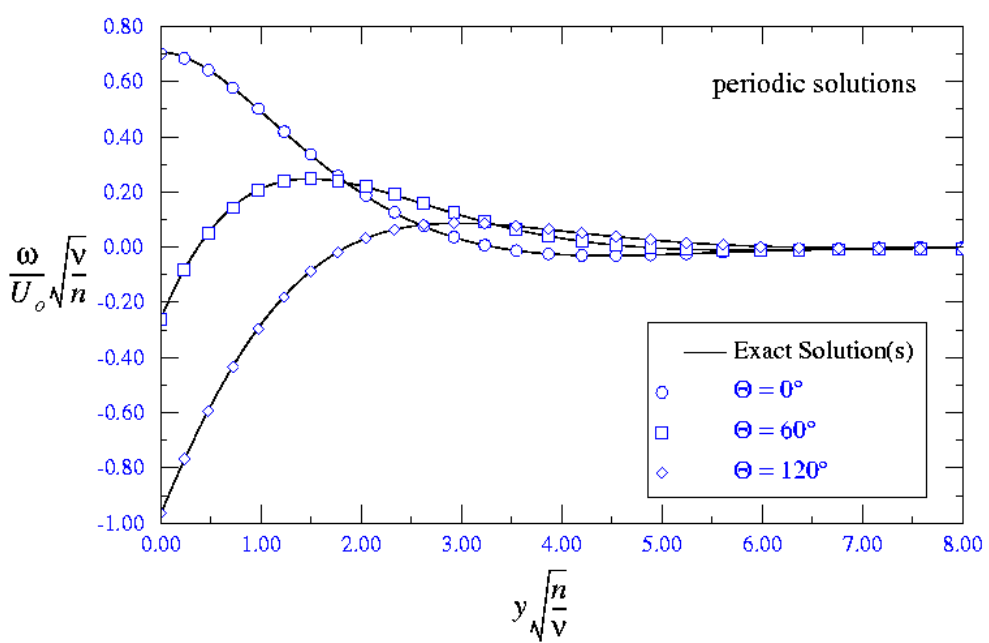

Figure 9. Vorticity Distributions for Periodic Solutions of Sinusoidally Oscillating Plate 


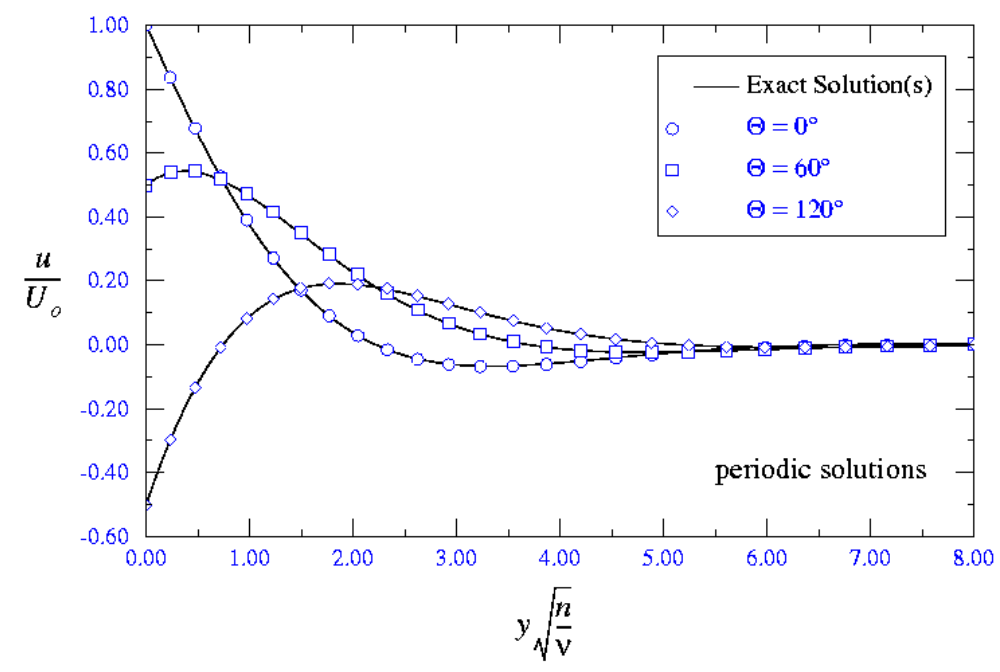

Figure 10. Tangential Velocity Distributions for Periodic Solutions of Sinusoidally Oscillating Plate

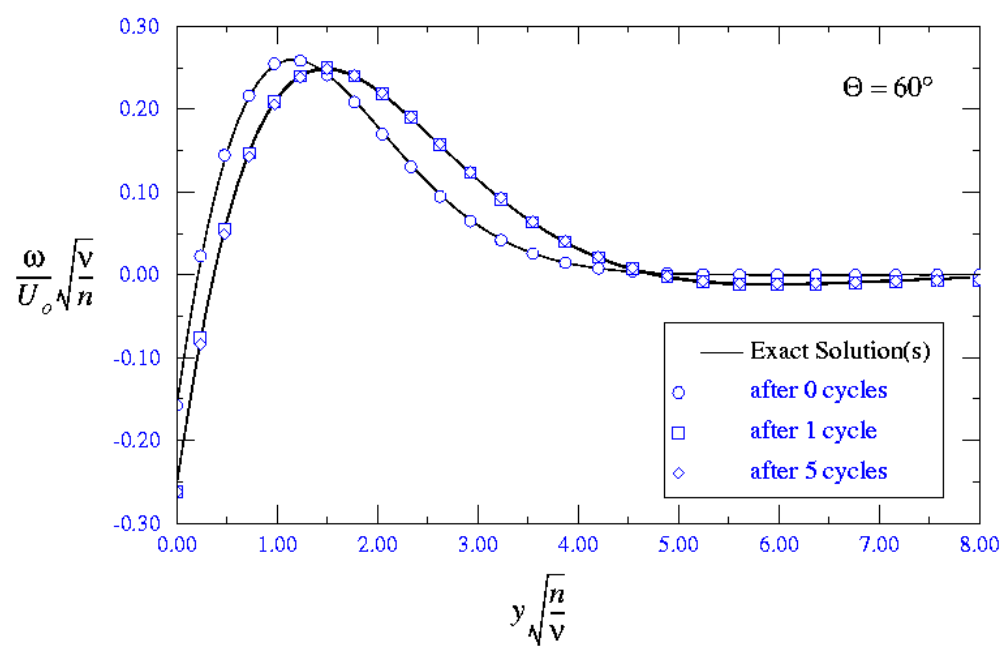

Figure 11. Vorticity Distributions for Transient Solutions of Sinusoidally Oscillating Plate 


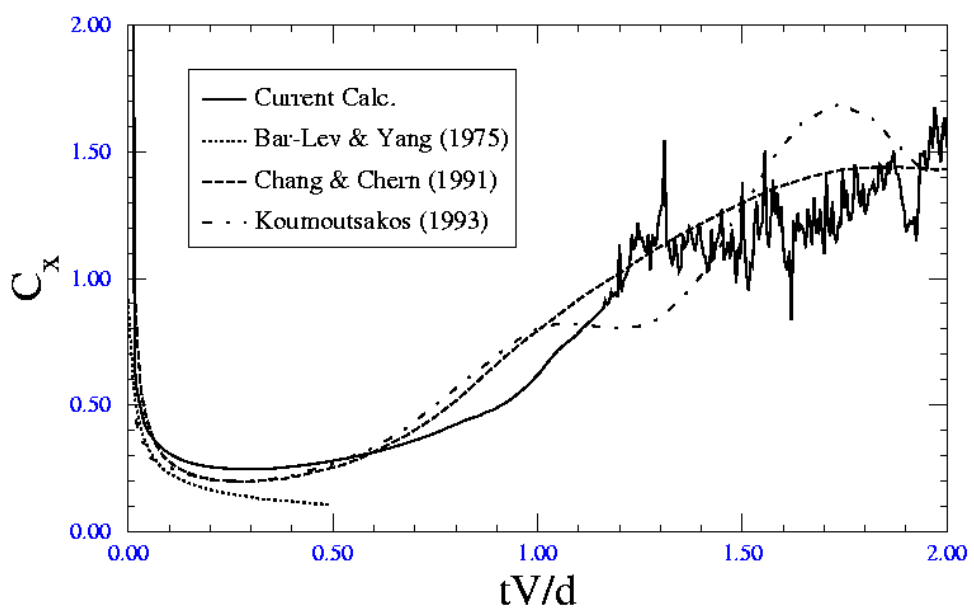

Figure 12. Comparisons of Drag Predictions for Impulsively Started Circular Cylinder $(\mathrm{Re}=$ 9500)

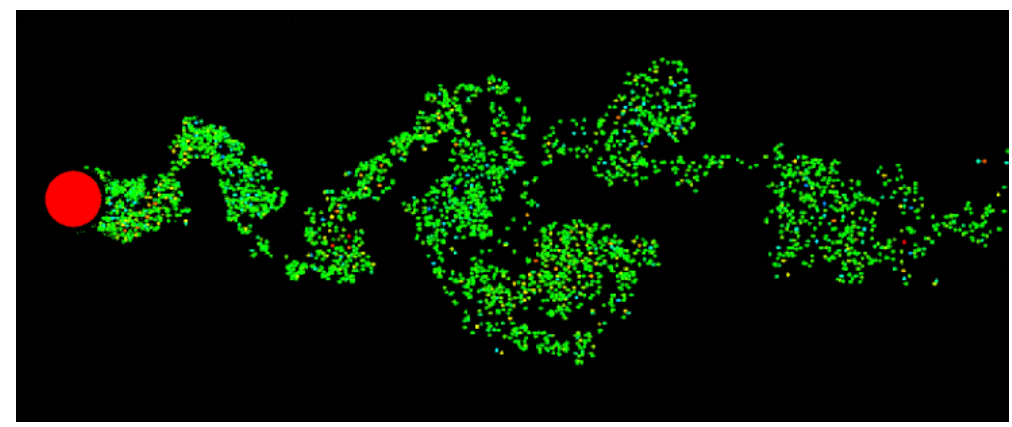

Figure 13. Calculated Wake Behind an Impulsively Started Circular Cylinder $(\operatorname{Re}=90,000)$

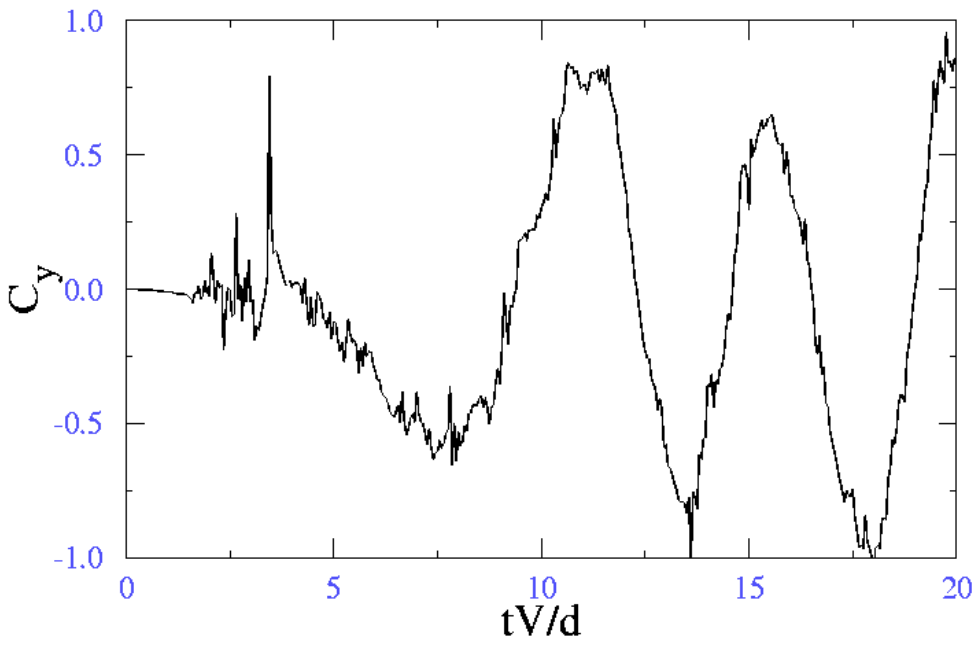

Figure 14. Side Force Coefficient for Impulsively Started Circular Cylinder $(\operatorname{Re}=\mathbf{9 0 , 0 0 0})$ 


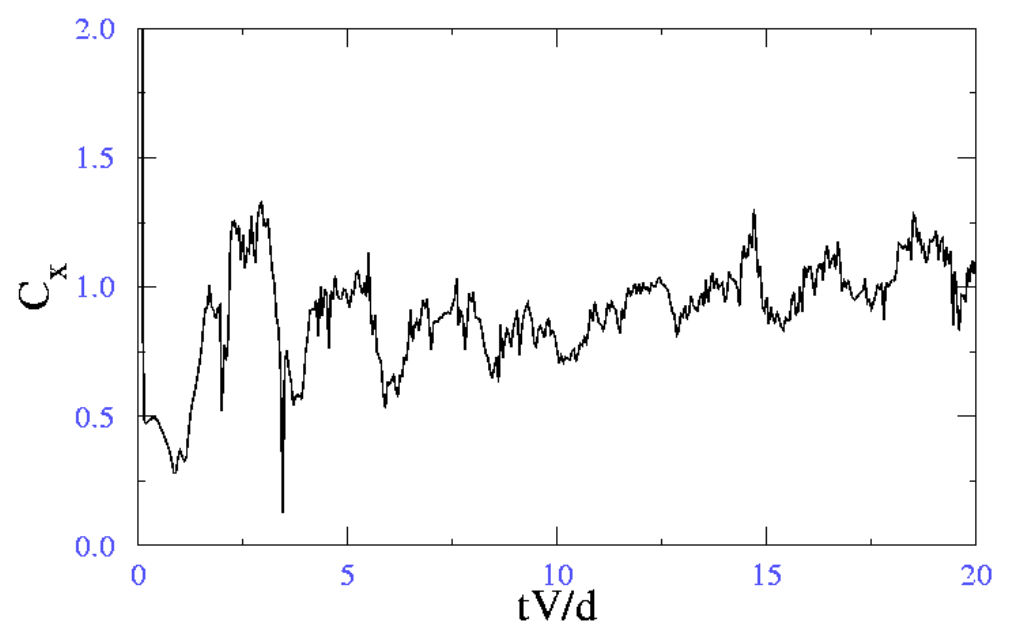

Figure 15. Drag Coefficient for Impulsively Started Circular Cylinder $(\operatorname{Re}=\mathbf{9 0 , 0 0 0})$ 